\title{
Target therapy for metastatic alveolar soft part sarcoma: a retrospective study with 47 cases
}

\author{
Jiayong Liu", Zhengfu Fan", Shu Li, Tian Gao, Ruifeng Xue, Chujie Bai, Lu Zhang, Zhichao Tan, \\ Zhiwei Fang
}

Department of Bone and Soft Tissue Tumor, Peking University Cancer Hospital, Beijing, China

Contributions: (I) Conception and design: J Liu, Z Fan; (II) Administrative support: Z Fang; (III) Provision of study materials or patients: R Xue, C Bai; (IV) Collection and assembly of data: L Zhang, Z Tan; (V) Data analysis and interpretation: S Li, T Gao; (VI) Manuscript writing: All authors; (VII)

Final approval of manuscript: All authors.

\#These authors contributed equally to this study.

Correspondence to: Jiayong Liu, MD. Key Laboratory of Carcinogenesis and Translational Research (Ministry of Education/Beijing), Department of Bone and Soft Tissue Tumor, Peking University Cancer Hospital \& Institute, 52, Fucheng Rd, Beijing, China. Email: liujiayong_doc@163.com.

Background: Alveolar soft part sarcoma (ASPS) is a translocation-associated soft-tissue tumor resistant to conventional cytotoxic agents. This report aims to compare the efficacy of anlotinib versus pazopanib as targeted monotherapy in metastatic ASPS and to determine the impact of drug dosage reduction on disease control.

Methods: Sixteen and 31 patients with metastatic ASPS were respectively treated with anlotinib and pazopanib monotherapy at a single institution. Objective response rate (ORR), progression-free survival (PFS), and overall survival (OS) were retrieved and compared between both therapeutic arms. Adverse events (AEs) within each group were recorded. Kaplan-Meier survivorship curves computed the impact of drug dosage reduction on PFS.

Results: The anlotinib group showed an ORR of $31.2 \%$, compared to $35.5 \%$ in the pazopanib arm $(\mathrm{P}=0.772)$. Median PFS was 23.6 months [95\% confidence interval (CI), 16.2-31.0 months] in patients treated with anlotinib, but dropped to 13.7 months (95\% CI, 10.8-16.7 months) in those managed with pazopanib $(\mathrm{P}=0.023)$. One (6.3\%) patient on anlotinib and 11 (35.5\%) on pazopanib developed AEs requiring drug dosage reduction ( $\mathrm{P}=0.029)$, which significantly reduced patients' $\mathrm{PFS}$ in the latter setting (10.5 vs. 15.8 months, $\mathrm{P}=0.012$ ). In patients without dosage reduction, anlotinib showed a bordering advantage than pazopanib on median PFS (24.5 vs. 15.8 months, $\mathrm{P}=0.112$ ).

Conclusions: Compared to pazopanib, anlotinib yielded longer PFS and lower incidence of AEs in ASPS patients. Drug dosage reduction was more frequently encountered with the former agent and affected the disease control.

Keywords: Alveolar soft part sarcoma (ASPS); anlotinib; pazopanib; objective response rate (ORR); progressionfree survival (PFS); adverse events (AEs)

Submitted Aug 25, 2020. Accepted for publication Nov 18, 2020.

doi: $10.21037 / \mathrm{atm}-20-6377$

View this article at: http://dx.doi.org/10.21037/atm-20-6377

\section{Introduction}

Alveolar soft part sarcoma (ASPS) is a rare, distinctive, and highly vascular neoplasm, which accounts for less than $1 \%$ of all soft-tissue sarcomas. It predominantly affects adolescents and young adults, and most commonly primarily occurs in the deep soft tissues in lower limbs, head and neck regions such as tongue and orbit (1). Despite being characterized by a relatively indolent clinical behavior, this disease carries an inferior prognosis, with up to $40 \%$ and $65 \%$ of patients presenting with distant metastasis at the 
time of diagnosis and during follow-up, respectively. The prognosis was influenced by the age of patients, the size of primary tumors and the metastatic status at diagnosis, with a median overall survival of around 11 years in patients without metastasis at diagnosis and 3 years in patients with metastatic disease (2-5).

Alveolar soft part sarcoma has a distinctive pseudoalveolar histological appearance and is highly vascularized with small vascular spaces separating nests of cells. The pseudoalveolar pattern appears to be due to necrosis of the centrally located cells in the nests. Cytogenetically, ASPS contains a characteristic $T(X ; 17)(\mathrm{p} 11 ; \mathrm{q} 25)$ translocation to form the ASPSCR1-TFE3 fusion gene, which can be detected by polymerase chain reaction or fluorescence in situ hybridization $(6,7)$. The transcriptional fusion protein acts as an abnormal transcription factor that can drive MET signaling and up-regulate transcripts related to angiogenesis, cell proliferation and metastasis (3). The expression of ASPSCR1-TFE3 gene can also induce upregulation of pro-inflammatory cytokines IL-1, IL-6 and IL-8, so as to promote the formation of pro-tumor microenvironment, including induction of proliferation, epithelial to mesenchymal transformation, tumor invasion and angiogenesis (8).

Standard cytotoxic chemotherapy, commonly used for soft-tissue sarcoma (STS), has a limited therapeutic value in ASPS (9-11). Multi-receptor tyrosine kinase inhibitors, including sunitinib (12,13), pazopanib (14-16), and cediranib $(17,18)$, showed objective responses in clinical trials involving ASPS patients with advanced or metastatic diseases. However, due to the low incidence of ASPS and the small number of cases in clinical studies, the factors associated with the effectiveness of targeted therapy remain unclear. Although most multi-receptor TKIs have a high response rate to ASPS, there are some differences in the effects and adverse events between different agents. For example, pazopanib which has been widely used since its approval in 2012 by the FDA to treat STS, albeit associated with several potentially lethal adverse events (AEs) (19-21). Not all patients can tolerate the standard dosage of $800 \mathrm{mg} / \mathrm{day}$ and need dose reductions during treatment. In an integral analysis of prospective clinical trials, the median dose intensity of pazopanib was estimated as $591 \mathrm{mg} /$ day (15), or approx. $75 \%$ of the standard dose. Anlotinib, another multi-receptor TKI, has been well tolerated in a phase II clinical trials for STS and shown to be highly effective in ASPS, with an objective response rate (ORR) of $46.2 \%$ and a median progression-free survival (PFS) of 21 months (22).
Because of their excellent response to TKIs, patients with ASPS often required prolonged time of medication. Therefore, it is important to choose an appropriate dosage of the target agents in order to ensure the continuity of treatment as well as to maintain the therapeutic effect. There is also a clinical report proposing that a reduced dose of pazopanib could be more beneficial for STS patients (23). However, there is still a lack of research on the appropriate dose of pazopanib, especially in Asian populations. And whether other TKIs, such as anlotinib, have comparable effect as pazopanib is not clear. We thus conducted the present study to analyse the effect and safety in metastatic ASPS patients receiving either anlotinib or pazopanib monotherapy in a single institution. We also investigated the proportion of dosage reduction required in the two therapeutic groups and the impact of a dosage reduction on disease control.

We present the following article in accordance with the STROBE reporting checklist (available at http://dx.doi. org/10.21037/atm-20-6377).

\section{Methods}

This retrospective study was undertaken at a single institution and was approved by the Ethics Committee of Peking University Cancer Hospital (Clinical trial registration number: 2013042210). The study was conducted in accordance with the Declaration of Helsinki (as revised in 2013). We obtained written, informed consent from all patients.

Between December 2012 and March 2017, 47 patients with metastatic ASPS treated with either anlotinib (group A) or pazopanib (group B) were identified from our institutional database and assessed for eligibility. Experienced pathologists set up histological diagnosis. All patients had received no prior anti-angiogenic treatment and had an Eastern Cooperative Oncology Group (ECOG) performance status score of $\leq 1$ at the time of presentation, with no evidence of uncontrolled hypertension (blood pressure $>150 / 90 \mathrm{mmHg}$ despite adequate therapy), bone marrow depression, coagulation disorder, or liver/renal dysfunction. We excluded patients with brain metastasis or tumors amenable to surgery at diagnosis.

Twenty-two patients with ASPS were enrolled in a series of clinical trials on the treatment of soft tissue sarcoma with anlotinib (NCT01878448 and NCT02449343). Six patients who received a placebo were excluded. We assigned the remaining 16 individuals to group A and treated with $12 \mathrm{mg}$ 
Table 1 Patient characteristics

\begin{tabular}{lccc}
\hline Characteristics & Anlotinib $(\mathrm{n}=16)$ & Pazopanib $(\mathrm{n}=31)$ & $\mathrm{P}$ value \\
\hline Sex, $\mathrm{n}(\%)$ & $7(43.8)$ & $10(32.3)$ & 0.53 \\
Male & $9(56.2)$ & $21(67.7)$ & \\
Female & 31 & 26 & 0.29 \\
Age (year), median & 63 & 61 & 0.72 \\
Weight (kg), median & & & 0.61 \\
ECOG status, n (\%) & $11(68.8)$ & $19(61.3)$ & \\
0 & $5(31.2)$ & $12(38.7)$ & \\
1 & & & \\
Organs involved & & & \\
Lung & 16 & 29 & 0.54 \\
Bone & 4 & 9 & 0.69 \\
Liver & 2 & 7 & 0.75 \\
Others & $6(50.0)$ & $14(45.2)$ & \\
Prior chemotherapy, $\mathrm{n}(\%)$ & & 10 & \\
Yes & $8(50.0)$ & & \\
No & & & \\
\hline
\end{tabular}

${ }^{\dagger}$, numbers do not add up along this row due to some patients having more than one metastatic site. ECOG, Eastern Cooperative Oncology Group.

of anlotinib daily, 1 hour before breakfast, for the first 14 days in the 21-day cycle. Group B included 31 patients given pazopanib in ordinary outpatient practice at an initial dose of 600 or $800 \mathrm{mg}(800 \mathrm{mg}$ for patients with body weight $>80 \mathrm{~kg}$ ) once daily, 1 hour before or 2 hours after meals. Doses were reduced to $10 \mathrm{mg}$ for anlotinib and 400 to $600 \mathrm{mg}$ for pazopanib upon the emergence of AEs that affected the patients' usual daily activity. Treatment was continued until disease progression, incapacitating AEs, or death. Computer tomography (CT) and magnetic resonance imaging (MRI) are performed at baseline, and every 6 or 8 weeks for the anlotinib or pazopanib groups, respectively. History and physical examination, complete blood counts, serum chemistry, and thyroid function tests are performed at baseline in both therapeutic arms. Patients receiving anlotinib also had the same tests conducted weekly for the first cycle and every three weeks after that, while individuals treated with pazopanib had these tests performed at 4-week intervals. We asked all patients to monitor and record their blood pressure at home twice a day.
The primary endpoints of this study comprised the ORR, PFS, OS, and AEs. Tumor response was evaluated according to the Response Evaluation Criteria In Solid Tumors (RECIST), version 1.1 (24). At the time of the primary analysis, the best overall response was recorded. ORR is defined as the combined proportion of complete (CR) and partial responses (PR). The time interval between study enrollment and first disease progression or death was labeled PFS and was censored at the last time point at which the patient was considered progression-free. The OS was defined as the time from registration to death from any cause and was completed at the last contact date for living patients. AEs were graded according to a descriptive scale within the National Cancer Institute's Common Terminology Criteria for Adverse Events (NCI-CTCAE) (25). Patient data were extracted from electronic medical records and imaging studies.

\section{Statistics}

Within each therapeutic arm, frequencies, means, and standard deviations were used to describe the tendency and distribution of the different parameters, including ORR and AEs. Intergroup comparisons of ORR were performed with the Pearson Chi-square $\left(\chi^{2}\right)$ test. The effect of drug dosage reduction on Kaplan-Meier survival curves was determined using the log-rank test. A $\mathrm{P}$ value less than 0.05 was considered statistically significant. All analyses were performed with SPSS $^{\circledR}$ software version 24.0 (IBM, Armonk, New York, USA).

\section{Results}

\section{Patient characteristics}

This study sample comprised 47 patients with histologically proven metastatic ASPS treated with either anlotinib (16 patients) or pazopanib (31 individuals). There were $17(36.2 \%)$ males and $30(63.8 \%)$ females. The mean age was 27.8 years (range, 15-49 years), and the mean weight was $62.1 \mathrm{~kg}$ (range, $47-88 \mathrm{~kg}$ ). In all cases, performance status (ECOG) was $\leq 1$. The lung was the most frequently documented site of metastasis, with 45 (95.7\%) patients affected. Prior anthracycline-based chemotherapy was administered to $22(46.8 \%)$ individuals. No association was found between patients of each therapeutic group $(\mathrm{P}>0.05$, Table 1). 
Table 2 Responses to anlotinib or pazopanib according to the RECIST criteria

\begin{tabular}{lcccccc}
\hline Drug & CR $(\%)$ & PR $(\%)$ & SD $(\%)$ & PD (\%) & ORR (\%) & DCR (\%) \\
\hline Anlotinib $(n=16)$ & 0 & $5(31.2)$ & $10(62.5)$ & $1(6.3)$ & $5(31.2)$ & $15(93.7)$ \\
Pazopanib $(n=31)$ & $1(3.2)$ & $10(29.0)$ & $17(58.1)$ & $3(9.7)$ & $11(35.5)$ & $23(90.3)$ \\
Total $(n=47)$ & $1(2.1)$ & $15(31.9)$ & $27(57.4)$ & $4(8.5)$ & $16(34.0)$ & $43(91.2)$ \\
\hline
\end{tabular}

CR, complete response; PR, partial response; SD, stable disease; PD, progressive disease; ORR, objective response rate; DCR, disease control rate.

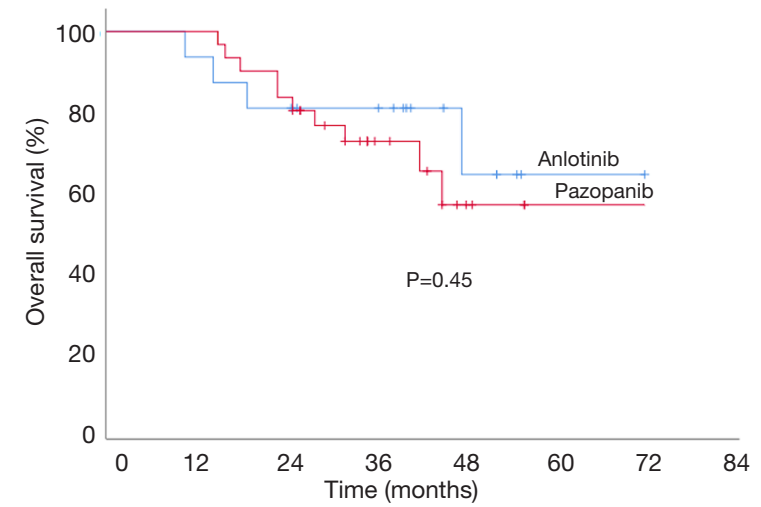

Figure 1 OS rates of patients in both therapeutic arms. OS, overall survival.

\section{Response}

All patients in group A received at least four cycles (12 weeks) of anlotinib. The best responses to anlotinib included 5 PR (31.3\%) and $10 \mathrm{SD}$ (Table 2). In group $\mathrm{B}$, pazopanib was discontinued in 1 patient because of pneumothorax after three weeks, when the CT scan revealed a slight reduction in lung lesions (SD), 30 patients (which received at least eight weeks of pazopanib, regardless of their initial drug dosage ( 25 patients were administered $600 \mathrm{mg} /$ day and five individuals started on an $800 \mathrm{mg} /$ day regimen). In one patient, the lesions in the lung completely response (CR) after five months of pazopanib, and she discontinued the drug for economic reasons. Although pulmonary recurrence was found in 4 months after discontinuation, she observed and then enrolled in a clinical trial of PD1 monoclonal antibody. Of all 31 patients in group $\mathrm{B}$, they showed an ORR of $35.5 \%$, the best responses to pazopanib encompassed $1 \mathrm{CR}, 10 \mathrm{PR}$, and $17 \mathrm{SD}$ (Table 2). ORR does not statistically differ between the two therapeutic arms $(\mathrm{P}=0.77$ and $\mathrm{P}=0.69$, respectively).

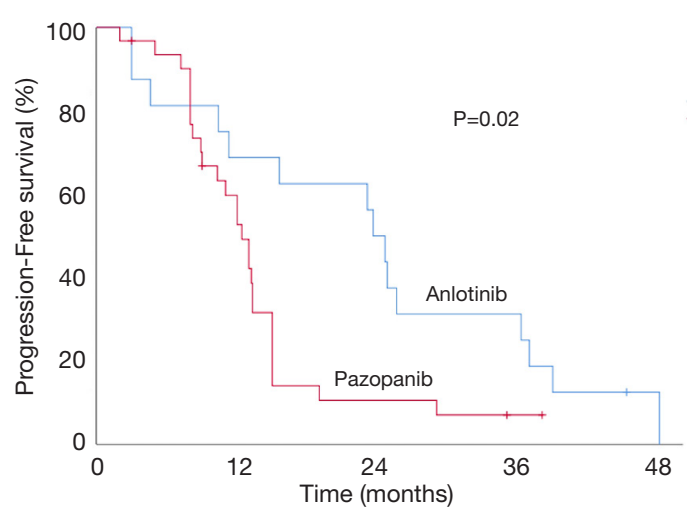

Figure 2 PFS rates of patients in both therapeutic groups. PFS, progression-free survival.

\section{Survival}

The median follow-ups were 36.8 and 35.6 months for the anlotinib and pazopanib groups, respectively. Although the median OS was not reached in either therapeutic arm at the time of the analysis, the estimated cumulative OS did not significantly differ between patients on anlotinib (57.3 months) or pazopanib (54.4 months) ( $\mathrm{P}=0.45$, Figure 1).

The median PFS in group A (anlotinib) was 23.6 months [95\% confidence interval (CI), 16.2-31.0 months], with $1(6.25 \%)$ patients still on medication at their last followup. In group B (pazopanib), median PFS was reduced to 13.7 months (95\% CI, 10.8-16.7 months), with 2 (6.45\%) patients still receiving therapy at last follow-up. Patients receiving anlotinib exhibited a longer PFS compared to those on pazopanib ( $\mathrm{P}=0.02$, Figure 2).

\section{AEs}

The most common AEs in patients treated with anlotinib included hypothyroidism (15 patients, 93.4\%), hand-foot 
Table 3 Dosage reduction characteristics

\begin{tabular}{|c|c|c|c|c|c|c|c|c|}
\hline No. & Drug & Gender & Age (year) & Initial dose $(\mathrm{mg})$ & Adjusted dose (mg) & Time to reduction (weeks) & Associated AEs & PFS (months) \\
\hline 1 & $A$ & M & 26 & 12 & 10 & 6 & QT prolongation & 10.4 \\
\hline 2 & $\mathrm{P}$ & M & 33 & 600 & 400 & 3 & Hypertension & 11.2 \\
\hline 3 & $\mathrm{P}$ & M & 23 & 600 & 400 & 10 & Diarrhea & 10.3 \\
\hline 4 & $\mathrm{P}$ & M & 26 & 800 & 600 & 3 & Hypertension & 12.4 \\
\hline 5 & $\mathrm{P}$ & $\mathrm{F}$ & 18 & 600 & 400 & 6 & Hand-foot reaction & 19.0 \\
\hline 6 & $\mathrm{P}$ & $\mathrm{F}$ & 16 & 600 & 400 & 5 & Elevated ALT/AST & 5.2 \\
\hline 7 & $P$ & $\mathrm{~F}$ & 25 & 600 & 400 & 6 & Hand-foot reaction & 12.8 \\
\hline 8 & $\mathrm{P}$ & M & 22 & 800 & 400 & 6 & Hand-foot reaction, diarrhea & 8.2 \\
\hline 9 & $P$ & M & 33 & 600 & 400 & 4 & Hypertension & 12.1 \\
\hline 10 & $\mathrm{P}$ & $\mathrm{F}$ & 19 & 600 & 400 & 6 & Hand-foot reaction & 8.2 \\
\hline 11 & $\mathrm{P}$ & $\mathrm{F}$ & 16 & 600 & 400 & 3 & Mucositis, diarrhea & 8.9 \\
\hline 12 & $\mathrm{P}$ & $\mathrm{F}$ & 25 & 800 & 600 & 4 & Hypertension & 7.2 \\
\hline
\end{tabular}

AEs, adverse events; PFS, progression-free survival; A, anlotinib; M, male; P, pazopanib; F, female.

syndrome (12 patients, $75 \%$ ), diarrhea (10 patients, $62.5 \%$ ), hypertension, and oral mucositis (8 patients each, $50 \%$ ). They were all grades 1 or 2 , except $3(18.8 \%)$ patients with grade 3 hypertension, 1 (6.3\%) patient with grade 3 diarrhea, and $2(12.5 \%)$ patients with grade 3 liver enzymes' elevation ( $\gamma$-glutamyl transpeptidase, GGT). One $(6.3 \%)$ patient developed a grade 3 prolonged QT interval on the electrocardiogram. During the therapeutic course, 7 (43.8\%) individuals presented with grade 3 toxicities, but no grade 4 AEs were documented.

In the pazopanib group, 25 and 6 patients received an initial dose of 600 and $800 \mathrm{mg}$, respectively. Thirty patients continued therapy for at least eight weeks. One patient developed pneumothorax after three weeks of medication and led to discontinuation. Most frequently mild-to-moderate encountered toxicities encompassed hypopigmentation (30 patients, 96.8\%), hand-foot syndrome (25 patients, $80.6 \%$ ), hypertension (23 patients, $74.2 \%$ ), diarrhea, and mucositis (19 patients each, $61.3 \%$ ). Seven $(22.6 \%)$ patients showed a grade 3 hypertension, while grade 3 hand-foot syndrome or diarrhea was documented in 6 patients (19.4\%). Less common AEs included grade 3 elevated liver enzymes, mucositis, and fatigue (3 patients each, 9.7\%). Only 1 (3.2\%) grade 4 hypertension was found. 18 (58.1\%) patients complained of grades 3 or 4 toxicities during their treatment.

\section{Dosage reduction}

Twelve out of 47 (25.5\%) patients had a dosage reduction during their targeted therapy course (Table 3). One patient receiving anlotinib had a dose reduction from 12 to $10 \mathrm{mg}$ per day from a prolonged QT interval on an electrocardiogram despite the absence of relevant clinical symptoms, and he remained at a stable disease for 10.4 months. In the pazopanib group, 11 patients had their medication dose reduced. Three individuals with an initial dose of $800 \mathrm{mg}$ per day switched to either $600 \mathrm{mg}$ (2 patients) or $400 \mathrm{mg}$ (1 patient). Eight added patients had their pazopanib dose decreased from 600 to $400 \mathrm{mg}$ per day. These reductions resulted from a hand-foot syndrome (4 cases), hypertension ( 4 cases), diarrhea (4 cases), mucositis ( 3 cases), and elevated ALT/AST ( 1 case). The median time from the first medication to dosage reduction was 5.5 weeks (3-10 weeks). Dosage reduction occurred more frequently in the pazopanib group (Pearson, $\mathrm{P}=0.03$ ), and KaplanMeier survival curves showed that in this group, the median PFS of patients with dosage reduction was significantly shorter than that without dosage reduction (10.5 vs. 15.8 months, $\mathrm{P}=0.01$, Figure 3). We also compared the median PFS of patients without dosage reduction between the two groups, and the results showed anlotinib had a bordering advantage than pazopanib (24.5 vs. 15.8 months, $\mathrm{P}=0.11$, Figure 4). 


\section{Page 6 of 9}

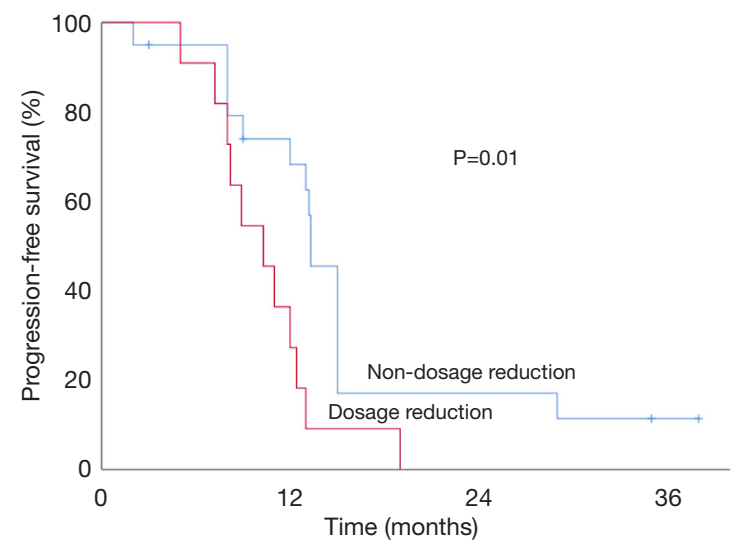

Figure 3 Impact of dosage reduction on PFS. PFS, progressionfree survival.

\section{Discussion}

ASPS is a rare soft-tissue malignancy with indolent biological behavior. Researchers have proved that although conventional cytotoxic regimens ineffective for this disease, studies assessing the efficacy of targeted therapy with multireceptor tyrosine kinase inhibitors were more promising $(12,17,26,27)$. Our retrospective study corroborates these results by documenting an ORR higher than $30 \%$ and a median PFS time over 13 months in both anlotinib and pazopanib therapeutic arms. The findings might be associated with the unique angiogenic signature of ASPS and the expression of its characteristic unbalanced ASPSCR1-TFE3 fusion $(7,28)$.

Although ORR did not statistically differ between anlotinib- and pazopanib-treated groups, the median PFS achieved with the former was significantly longer than the latter. After excluding the effect of dose reduction on PFS, we found that the median PFS of the anlotinib group was still bordering superior to the pazopanib group; this suggests the two agents may differ in their mechanisms of action. Even though preclinical studies have shown the target spectrum of pazopanib and anlotinib are similar (including VEGFR, PDGFR, FGFR, and c-Kit), there are some differences in their efficacy on different targets (29-31). The frequency and extend of AEs documented in this study also support this idea. All (96.8\%) patients on pazopanib experienced hair hypopigmentation compared to none of the anlotinib-treated patients. Hair hypopigmentation is associated with an interruption of the c-kit signaling pathway $(32,33)$. Further, it was observed to be more common in those with pazopanib than sunitinib. In this
Liu et al. Target therapy for metastatic alveolar soft part sarcoma

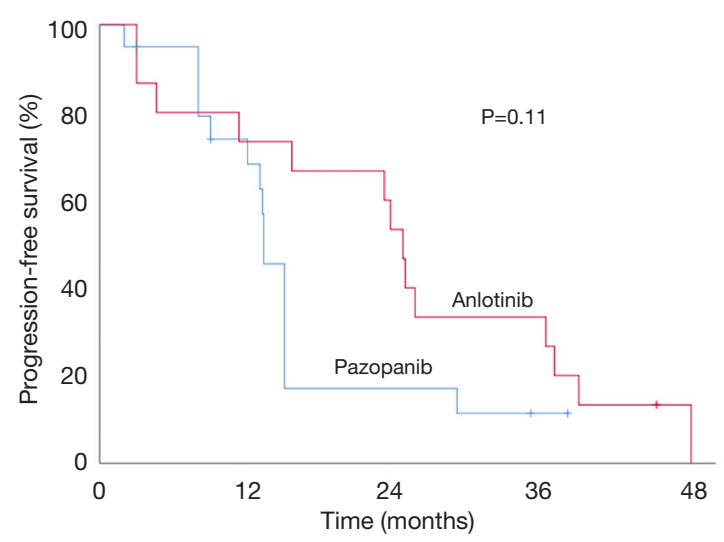

Figure 4 PFS rates of patients without dosage reduction in both therapeutic groups. PFS, progression-free survival.

study, there was no hair hypopigmentation in patients with anlotinib, indicating that it had little influence on the c-kit pathway.

It has been shown that anlotinib can suppress growth and metastasis vial blockade of the MET pathway other than VEGFs in vitro and in vivo models of human osteosarcoma (34). Gene expression profiling studies show the ASPSCR1-TFE3 fusion protein acts as an aberrant transcription factor that drives MET signaling and upregulation of the transcripts associated with angiogenesis, cell proliferation, and metastasis $(7,11)$. Further research is called for to elucidate the exact mechanism of anlotinib in the MET pathway in ASPS.

The incidence of AEs, grades 3 or 4 hypertension, hand-foot syndrome, and diarrhea-was higher in patients receiving pazopanib compared to those with anlotinib, regardless of the initial dosage of the former. In the PALETTE study, upon the results of which the FDA approved pazopanib as a therapeutic option for soft-tissue sarcomas, the mean daily dose of pazopanib was lower in the Japanese subgroup compared to the general population (624.4 vs. $700.4 \mathrm{mg}$, respectively) and the AEs leading to dose reduction was more frequently identified in the former (14). Our study corroborates these findings and shows the standard recommended dose of $800 \mathrm{mg}$ of pazopanib may not be suitable for Asian patients to start with it. Also, nine patients (29.0\%) were able to tolerate a dose of only $400 \mathrm{mg}$. The predominance might explain the phenomena of female $(67.7 \%)$ patients in the pazopanib group, with a median weight of $61 \mathrm{~kg}$, which may have affected dose reduction in this setting. 
In the pazopanib group, the median PFS was shorter in patients with dosage reduction than those without it. The decrease in blood concentration caused by drug reduction may be the reason for the shortening of PFS. Nakano reported that among the patients who achieved disease control with pazopanib, the PFS of those who received $>80 \%$ of standard dosage in the first 12 weeks was better than those who received $<80 \%$ standard dosage (35). Also, it may be related to the evaluation method. Following the RECIST standard, the criteria for determining PD are all on the minimum tumor shrinkage as the baseline. Patients with a high initial dose may obtain a relatively high remission rate in a short period, resulting in relatively apparent tumor shrinkage. However, the anti-tumor efficacy is reduced after drug dosage reduction, and the optimal remission status of the high-dose group as a reference is likely to affect the duration of PFS.

This study did not detect a statistical difference in OS between anlotinib and pazopanib groups, which were both not reaching the median OS. These results may be related to the short follow-up period despite both arms being followed for a median of 36 months. Multiple reports have documented favorable long-term survival even after the development of metastasis in patients with ASPS, which might be related to the indolent character of the disease $(2,11)$. In line with these results, most patients underwent other therapies with other anti-angiogenic agents (sunitinib, cediranib, or apatinib) or immune checkpoint inhibitors (anti-PD-1 antibody) after failure on anlotinib/pazopanib. These subsequent treatments have made the analysis of OS more difficult. It has been shown that the use of second-line TKIs still had a certain efficiency after resistance of firstline TKIs, due to the different spectrum of targets for each drug (28). For example, cediranib has been shown to affect multiple intracellular pathways, such as ANGPT2, FLT1, and KDR, as well as MAPK (16). In addition, immune checkpoint inhibitors (ICIs), such as pembrolizumab, has represented a promising area of drug development in ASPS (36). Furthermore, some investigators have explored a more efficient response by combination of checkpoint inhibitors with TKIs in ASPS. In a phase 2 study examining axitinib combined with pembrolizumab in STS, among 11 patients with ASPS evaluable for response, 7 patients achieved PR and 3 patients had SD (37).

This study is a retrospective analysis that reports the experience of a single institute in the management of a small cohort of metastatic ASPS patients. Limited by the rarity of this disease, designing a prospective clinical trial to include a substantial number of patients is challenging without involving multiple institutions with several resulting therapeutic modalities and regimens. In order to minimize the bias in this retrospective study, all subjects in the present study were managed with identical therapeutic regimens (anlotinib or pazopanib monotherapy) by the same medical teams to ensure our results were relevant to and applicable in current clinical practice. And because most of the patients in this study were young and had a good performance status, the treatment compliance was good and follow-up and examinations were regularly completed during treatment. Imaging evaluations were performed by independent radiologists, and all imaging and survival data were obtained

\section{Conclusions}

ASPS is sensitive to anlotinib and pazopanib monotherapy, as shown by its high response rate to either agent. Compared to pazopanib, anlotinib yields longer PFS and lower incidence of grade 3 to 4 toxicities. Patients on pazopanib were more prone to having their dosage reduced, and this affected the control of their disease.

\section{Acknowledgments}

Funding: Supported by the National Natural Science Foundation of China (Grant No. 81802689).

\section{Footnote}

Reporting Checklist: The authors have completed the STROBE reporting checklist. Available at http://dx.doi. org/10.21037/atm-20-6377

Data Sharing Statement: Available at http://dx.doi. org/10.21037/atm-20-6377

Conflicts of Interest: All authors have completed the ICMJE uniform disclosure form (available at http://dx.doi. org/10.21037/atm-20-6377). The authors have no conflicts of interest to declare.

Ethical Statement: The authors are accountable for all aspects of the work in ensuring that questions related to the accuracy or integrity of any part of the work are appropriately investigated and resolved. This retrospective study was undertaken at a single institution and was 


\section{Page 8 of 9}

approved by the Ethics Committee of Peking University Cancer Hospital (Clinical trial registration number: 2013042210). The study was conducted in accordance with the Declaration of Helsinki (as revised in 2013). We obtained written, informed consent from all patients.

Open Access Statement: This is an Open Access article distributed in accordance with the Creative Commons Attribution-NonCommercial-NoDerivs 4.0 International License (CC BY-NC-ND 4.0), which permits the noncommercial replication and distribution of the article with the strict proviso that no changes or edits are made and the original work is properly cited (including links to both the formal publication through the relevant DOI and the license). See: https://creativecommons.org/licenses/by-nc-nd/4.0/.

\section{References}

1. Zarrin-Khameh N, Kaye KS. Alveolar soft part sarcoma. Arch Pathol Lab Med 2007;131:488-91.

2. Portera CA Jr, Ho V, Patel SR, et al. Alveolar soft part sarcoma: clinical course and patterns of metastasis in 70 patients treated at a single institution. Cancer 2001;91:585-91.

3. Pennacchioli E, Fiore M, Collini P, et al. Alveolar soft part sarcoma: clinical presentation, treatment, and outcome in a series of 33 patients at a single institution. Ann Surg Oncol 2010;17:3229-33.

4. Ogura K, Beppu Y, Chuman H, et al. Alveolar soft part sarcoma: a single-center 26-patient case series and review of the literature. Sarcoma 2012;2012:907179.

5. Liu YP, Jin J, Wang WH, et al. A retrospective analysis of lung metastasis in 64 patients with alveolar soft part sarcoma. Clin Transl Oncol 2015;17:803-9.

6. Ladanyi M, Lui MY, Antonescu CR, et al. The der(17) $\mathrm{t}(\mathrm{X} ; 17)(\mathrm{p} 11 ; \mathrm{q} 25)$ of human alveolar soft part sarcoma fuses the TFE3 transcription factor gene to ASPL, a novel gene at 17q25. Oncogene 2001;20:48-57.

7. Stockwin LH, Vistica DT, Kenney S, et al. Gene expression profiling of alveolar soft-part sarcoma (ASPS). BMC Cancer 2009;9:22.

8. Ishiguro N, Yoshida H. ASPL-TFE3 Oncoprotein Regulates Cell Cycle Progression and Induces Cellular Senescence by Up-Regulating p21. Neoplasia 2016;18:626-35.

9. Ogose A, Yazawa Y, Ueda T, et al. Alveolar soft part sarcoma in Japan: multi-institutional study of 57 patients from the Japanese Musculoskeletal Oncology Group.

\section{Liu et al. Target therapy for metastatic alveolar soft part sarcoma}

Oncology 2003;65:7-13.

10. Reichardt P, Lindner T, Pink D, et al. Chemotherapy in alveolar soft part sarcomas. What do we know? Eur J Cancer 2003;39:1511-6.

11. Paoluzzi L, Maki RG. Diagnosis, Prognosis, and Treatment of Alveolar Soft-Part Sarcoma: A Review. JAMA Oncol 2019;5:254-60.

12. Stacchiotti S, Negri T, Zaffaroni N, et al. Sunitinib in advanced alveolar soft part sarcoma: evidence of a direct antitumor effect. Ann Oncol 2011;22:1682-90.

13. Ghose A, Tariq Z, Veltri S. Treatment of multidrug resistant advanced alveolar soft part sarcoma with sunitinib. Am J Ther 2012;19:e56-8.

14. van der Graaf WT, Blay JY, Chawla SP, et al. Pazopanib for metastatic soft-tissue sarcoma (PALETTE): a randomised, double-blind, placebo-controlled phase 3 trial. Lancet 2012;379:1879-86.

15. Kasper B, Sleijfer S, Litiere S, et al. Long-term responders and survivors on pazopanib for advanced soft tissue sarcomas: subanalysis of two European Organisation for Research and Treatment of Cancer (EORTC) clinical trials 62043 and 62072. Ann Oncol 2014;25:719-24.

16. Coens C, van der Graaf WT, Blay JY, et al. Health-related quality-of-life results from PALETTE: A randomized, double-blind, phase 3 trial of pazopanib versus placebo in patients with soft tissue sarcoma whose disease has progressed during or after prior chemotherapy-a European Organization for research and treatment of cancer soft tissue and bone sarcoma group global network study (EORTC 62072). Cancer 2015;121:2933-41.

17. Kummar S, Allen D, Monks A, et al. Cediranib for metastatic alveolar soft part sarcoma. J Clin Oncol 2013;31:2296-302.

18. Judson I, Scurr M, Gardner K, et al. Phase II study of cediranib in patients with advanced gastrointestinal stromal tumors or soft-tissue sarcoma. Clin Cancer Res 2014;20:3603-12.

19. Kapadia S, Hapani S, Choueiri TK, et al. Risk of liver toxicity with the angiogenesis inhibitor pazopanib in cancer patients. Acta Oncol 2013;52:1202-12.

20. Kobayashi E, Koyama T, Kobayashi K, et al. Reversible hair depigmentation in a Japanese female treated with pazopanib. J Dermatol 2014;41:1021-2.

21. Verschoor AJ, Gelderblom H. Pneumothorax as adverse event in patients with lung metastases of soft tissue sarcoma treated with pazopanib: a single reference centre case series. Clin Sarcoma Res 2014;4:14.

22. Chi Y, Fang Z, Hong X, et al. Safety and Efficacy of 
Anlotinib, a Multikinase Angiogenesis Inhibitor, in Patients with Refractory Metastatic Soft-Tissue Sarcoma. Clin Cancer Res 2018;24:5233-8.

23. Abe K, Yamamoto N, Hayashi K, et al. Balancing Prolonged Survival with QoL Using Low-dose Pazopanib Maintenance: A Comparison with the PALETTE Study. Anticancer Res 2016;36:2893-7.

24. Eisenhauer EA, Therasse P, Bogaerts J, et al. New response evaluation criteria in solid tumours: revised RECIST guideline (version 1.1). Eur J Cancer 2009;45:228-47.

25. US Department of Health and Human Services, National Institutes of Health, Institute. NC. Common Terminology Criteria for Adverse Events (CTCAE) Version 5.0. 2017. https://ctep.cancer.gov/protocoldevelopment/electronic_ applications/docs/CTCAE_v5_Quick_Reference_8.5x11. pdf. Accessed August 15, 2017.

26. Stacchiotti S, Mir O, Le Cesne A, et al. Activity of Pazopanib and Trabectedin in Advanced Alveolar Soft Part Sarcoma. Oncologist 2018;23:62-70.

27. Schöffski P, Wozniak A, Kasper B, et al. Activity and safety of crizotinib in patients with alveolar soft part sarcoma with rearrangement of TFE3: European Organization for Research and Treatment of Cancer (EORTC) phase II trial 90101 'CREATE'. Ann Oncol 2018;29:758-65.

28. Lazar AJ, Das P, Tuvin D, et al. Angiogenesis-promoting gene patterns in alveolar soft part sarcoma. Clin Cancer Res 2007;13:7314-21.

29. Lin B, Song X, Yang D, et al. Anlotinib inhibits angiogenesis via suppressing the activation of VEGFR2, PDGFRbeta and FGFR1. Gene 2018;654:77-86.

30. Zhang Z, Zhao Y, Lu F, et al. Multi-targeted tyrosine

Cite this article as: Liu J, Fan Z, Li S, Gao T, Xue R, Bai C, Zhang L, Tan Z, Fang Z. Target therapy for metastatic alveolar soft part sarcoma: a retrospective study with 47 cases. Ann Transl Med 2020;8(22):1493. doi: 10.21037/atm-20-6377 kinase inhibitors as third-line regimen in advanced nonsmall cell lung cancer: a network meta-analysis. Ann Transl Med 2019;7:452.

31. Stacchiotti S, Mir O, Le Cesne A, et al. Activity of Pazopanib and Trabectedin in Advanced Alveolar Soft Part Sarcoma. Oncologist 2018;23:62-70.

32. Routhouska S, Gilliam AC, Mirmirani P. Hair depigmentation during chemotherapy with a class III/ $\mathrm{V}$ receptor tyrosine kinase inhibitor. Arch Dermatol 2006;142:1477-9.

33. Motzer RJ, Hutson TE, Cella D, et al. Pazopanib versus sunitinib in metastatic renal-cell carcinoma. N Engl J Med 2013;369:722-31.

34. Wang G, Sun M, Jiang Y, et al. Anlotinib, a novel small molecular tyrosine kinase inhibitor, suppresses growth and metastasis via dual blockade of VEGFR2 and MET in osteosarcoma. Int J Cancer 2019;145:979-93.

35. Nakano K, Funauchi Y, Hayakawa K, et al. Relative Dose Intensity of Induction-Phase Pazopanib Treatment of Soft Tissue Sarcoma: Its Relationship with Prognoses of Pazopanib Responders. J Clin Med 2019;8:60.

36. Groisberg R, Hong DS, Behrang A, et al. Characteristics and outcomes of patients with advanced sarcoma enrolled in early phase immunotherapy trials. J Immunother Cancer 2017;5:100.

37. Wilky BA, Trucco MM, Subhawong TK, et al. Axitinib plus pembrolizumab in patients with advanced sarcomas including alveolar soft-part sarcoma: a single-centre, single-arm, phase 2 trial. Lancet Oncol 2019;20:837-48.

(English Language Editor: J. Chapnick) 